\title{
Anadolu Miyosen Dönem Bovidlerinin Paleobiyocoğrafyası ve Paleoekolojisinin Değerlendirmesi
}

\author{
Ali TAŞ \\ Kırşehir Ahi Evran Üniversitesi, Fen-Edebiyat Fakültesi, Antropoloji Bölümü, Kırşehir \\ alitas4092.gs@gmail.com \\ ORCID ID: https://orcid.org/0000-0002-2066-6075 \\ Ahmet Cem ERKMAN \\ Kırşehir Ahi Evran Üniversitesi, Fen-Edebiyat Fakültesi, Antropoloji Bölümü, Kırşehir \\ acerkman@ahievran.edu.tr \\ ORCID ID: https://orcid.org/0000-0003-3382-1019
}

Araştırma Makalesi

DOI: $10.31592 /$ aeusbed.608029

Geliş Tarihi: 21.08.2019

Revize Tarihi: 28.01 .2020

Kabul Tarihi: 04.02.2020

\section{Atıf Bilgisi}

Taş, A. ve Erkman, A. C. (2020). Anadolu Miyosen Dönem Bovidlerinin paleobiyocoğrafyası ve paleoekolojisinin değerlendirmesi. Ahi Evran Üniversitesi Sosyal Bilimler Enstitüsü Dergisi, 6(1), 87-102.

\section{ÖZ}

Miyosen döneme tarihlendirilen Anadolu'da 22, Yunanistan'da 30 ve İran'da 2 formasyondan ele geçen bovidlere ait taksonomik bilgiler paleobiyocoğrafik ve paleoekolojik dinamiklerle birlikte bu çalışmada analiz edilmiştir. Anadolu-İran-Yunanistan biyocoğrafik bölgesindeki lokalitelerde ortak bulunan sekiz farklı bovid türü (Gazella cf. capricornis, Gazella sp., Miotragoceros sp.,Oioceros rothi, Palaeoreas sp., Prostrepsiceros houtumschindleri, Prostrepsiceros rotundicornis ve Tragoportax amalthea) bu bölgeler arasında büyük ölçüde paleoekolojik ve paleobiyocoğrafik benzerlikler bulunduğunu göstermektedir. Aynı zamanda Anadolu'nun kıtalar arası geçişte bir köprü görevini gördüğ̈ ve bunun sonucunda da söz konusu türlerin Anadolu'da ve Avrupa'da temsil edildikleri seviyelerden daha yukarı seviyelerde olduğu görülmüştür. Anadolu'da Vallesian döneme kadar sınırlı sayıda bovidae ailesine ait cins ve türler mevcutken, Geç Vallesian ve Erken Turolian ile birlikte bir artış söz konusudur. Birçok bovidae türünün açık otlak habitatlara daha iyi adapte olması Geç Miyosen dönemde bovidlerin hızlı bir şekilde çeşitlenmesine ve yetmiş yeni türün evrilmesine yol açmıştır. Anadolu'da bovidae cinslerinin ve türlerinin adapte olduğu paleoekolojik koşullar dikkate alınırsa, yer yer nemli ve yer yer kurak karakterdeki subtropik orman örtüsünün yanında yazın yeşil savanaların ve bazen de tamamen kuruyan step otlaklıkların yaygın olduğu anlaşılmaktadır. Geç Miyosen dönemde ise daha çok açık savana-mozaik tipi ekolojik ortamın yaygın olduğu bir coğrafya söz konusudur. Ancak bu dönem İran-Anadolu-Yunanistan arasınadaki biyocoğrafik bölgenin düşünülenin aksine daha kompleks bir yapıya sahip olduğuna işaret etmektedir.

Anahtar Kelimeler: Bovid, Anadolu, paleobiyocoğrafya, paleoekoloji.

\section{Evaluation of Paleobiography and Paleoecology of Anatolian Miocene Bovides}

\begin{abstract}
Taxonomic information belonging to bovids obtained from 22 formations in Anatolia, 30 formations in Greece and 2 formations in Iran dated to Miocene period has been analyzed together with paleobiogeographic and paleoecological dynamics in this study. Eight different bovids (Gazella cf. capricornis, Gazella sp., Miotragoceros sp., Oioceros rothi, Palaeoreas sp., Prostrepsiceros houtumschindler, Prostrepsiceros rotundicornis and Tragoportax amalthea), which are common to localities in Anatolia-Iran-Greece provine, show that there are largely paleoecological and paleobiogeographic similarities between these regions. At the same time, these species acted as a bridge in the transition between the cross-continents of Anatolia and as a result of this, It is seen that they were at higher levels than they are represented in Anatolia and Europe. Untill the Vallesian period, a limited number of genera and species belonging to the bovid family existed, while there was an increase with Late Vallesian and Early Turolian. Better adaptation of many bovid species to open grassland habitats led to the rapid diversification of bovid species in the Late Miocene and the evolution of seventy new genera. Considering the paleoenvironmental conditions in which bovid species and species are adapted in Anatolia, In addition to the subtropical forest cover, which is sometimes humid and sometimes dry, green savannah and sometimes completely dry steppe grasslands appear to be widespread. In the late Miocene period, there is a geography where an open savanna-mosaic type ecological environment is common. However, this period points out that the bioprovensy between Iran, Anatolia and Greece has a more complex structure contrary to what is believed.
\end{abstract}

Keywords: Bovid, Anatolia, paleobiogeography, paleoecology. 


\section{Giriş}

Avrasya ve Afrika omurgalı memeli faunasının birbirleri ile olan göç ilişkilerinin hangi yollardan yapıldığının anlaşılması paleontolojik çalışmalar için büyük bir önem arz etmektedir. $\mathrm{Bu}$ bağlamda, Neojen dönemden itibaren Asya, Afrika ve Avrupa kıtaları arasında yer alan Anadolu göç izlerinin sürüldüğü bir yarımada olarak önemini hala korumaktadır. Paleontolojik çalışmalardan ele geçen buluntulara ait bir kısım memeli faunasının Avrupa'dan doğuya doğru olan göçleri sebebiyle, Anadolu'da Avrupa'da temsil edildikleri seviyelerden daha yukarı kronolojik seviyelerde bulunmuşlardır (Tekkaya, 1974). Avrasya omurgalı faunasının hareketlerinin tespitinde gerek batıdan doğuya gerek doğudan batıya doğru olan faunal göçlerin büyük çoğunluğunun Anadolu kanalıyla gerçekleştiği görülmektedir. Bu nedenle Anadolu Tersiyer dönem biyostratigrafisi oldukça karışıktır. Bu stratigrafik ve paleocoğrafik karmaşa büyük oranda bovid fosillerin katkısı ile çözülebilir.

Fosil artiodactyller muhtemelen sinıflandırılmasi en zor memeliler takımıdır (Prothero ve Foss, 2007). Güncel materyallerde artiodactyllerin taksonomisi oldukça kolay olmasına karşın nesli tükenmiş artiodactyller söz konusu olduğunda birçok zorluklarla karşıllaş1lır. Geviş getiren ve getirmeyen ruminantların yanında ruminant olmayanlar olarak alt bölümlere ayrılması nesli tükenmiş artiodactyller için hiç de kolay bir ölçüt değildir. Nesli tükenmiş artiodactyllerin sayısız ailesi bu listeye eklendiğinde birçok ailenin mantıklı ve uygun gruplanması için daha yüksek bir sinıflandırmanın gerekli olduğu anlaşılmaktadır (Colbert ve Moralles, 1991; Prothero ve Foss, 2007; Prothero ve Schoch, 2002). Bovidler, ruminantların en çeşitli grubudur. Güney Amerika, Avustralya dışındaki diğer kıtalarda ve doğal olarak farklı habitatlarda bulunurlar. Bovidler derin yağmur ormanlarından ormanlık alanlara, savanalara, çöllere, dağlara ve yapay ortamlara kadar birçok habitatta yaşarlar. Büyük ve küçük çeşitli formları mevcuttur. Yaklaşık olarak nesli tükenmiş 158 ve günümüzde yaşayan 47 adet klad vardır (Prothero ve Foss, 2007). Böylesine zengin bir yaşam alanı ve çeşitlilik derecesine sahip olan bovidler herbivor çalışmaları ve çeşitli iklim koşullarına uyum modellemeleri için ideal bir gurubu meydana getirirler. Paleobiyocoğrafya içinde iklimsel koşullar son derece büyük önem taşımaktadır. Erken ve Geç Neojen dönem boyunca kısa ve uzun vadeli büyük çevresel ve iklimsel değişimlerin yanında vejetasyondaki değişimler bovid türlerinin dağılımını ve farklılaşmasını şekillendirmiştir.

Bovid sistematiğinin en karakteristik özelliği boynuzlardır ve çoğunlukla fosil çalışmalarında kolay bulunurlar. Farklı şekillere sahip boynuz morfolojisi ve boynuz çekirdeği nesli tükenmiş bovid türlerinin teşhisinde oldukça önemlidir. Boynuzlardan sonra sistematik olarak belirleyici en önemli ikinci karakter dişlerdir. Dişler sert yapıları nedeniyle en iyi korunan fosil materyalleridir. Herbivor memelilerin molar taç yüksekliği bilinen en iyi eko-metrik göstergelerdendir. Farklı diyetler ve dişler türler arasında birbirinden ayrı diş aşınma derecelerini oluştururlar. Bu bağlamda, bovid türleri arasındaki farklı diş yapılarının hypsodonti ortalaması dikkate alındığında yağış ile arasında güçlü bir coğrafik korelasyon olduğu görülür. Ayrıca kafatası yapısı, premaksilla ve iskelet yapıları bovid türlerindeki diğer farklılıklardandır ve oldukça ayırt edici özellikler olup paleontolojik çalışmaların en önemli materyallerindendir (Fortelius, Eronen, Kaya, Tang, Raia, Puolamaki, 2014; Prothero ve Foss, 2007).

\section{Yöntem}

Miyosen döneme tarihlendirilen Anadolu'da 22, Yunanistan'da 30 ve İran'da 2 formasyondan ele geçen bovid türlerine ait literatürde yer alan taksonomik verileri paleobiyocoğrafik ve paleoekolojik veriler ile birlikte biostratigrafik analizi yapılmıştır. Toplanan veriler $\mathrm{MN}$ (Mammal Neogene) zonları (Dam, Alcala, Alonso Zarza, Calvo, Garces, Krijgsman, 2001) dikkate alınarak kronolojik sıraya konulmuştur. Paleo-çevresel koşulların tahmininde kullanılan NOW veri tabanına dayanan eko-metrik analiz yöntemi (Fortelius, Eronen, Jernvall, Pushkina, 2002; Fortelius vd., 2014) türlerin boynuz, cranial ve dental özellikler dikkate alınarak paleoekolojik verilerle birlikte üç bölge bazında korele edilmiştir. 


\section{Bulgular}

Neojen dönemden itibaren Anadolu Asya-Afrika-Avrupa kıtaları arasında bir köprü oluşturduğundan bovidae ailesinin göçlerinin izinin sürüldüğü bir yarımada olarak önemini korumaktadır. Türkiye'de Miyosen döneme ait birçok fosil buluntu yerleri mevcuttur ancak taksonomik bazda çalışılmış 22 lokalite dikkate alınmıştır (Tablo 1).

Tablo 1

Anadolu'da Bulunan Lokalitelere Ait Türler

\begin{tabular}{|c|c|c|c|c|}
\hline Lokalite & Bölge & $\mathrm{MN}$ & Tür & Araştırmacı \\
\hline \multirow{2}{*}{ Paşalar } & \multirow{2}{*}{ Bursa } & 6 & Capratragoides stehlini & \multirow{2}{*}{ Gentry, 1990} \\
\hline & & 7 & Hypsodontus pronaticornis & \\
\hline $\begin{array}{l}\text { Mordoğan-Ayıbalığı } \\
\text { mevkii }\end{array}$ & İzmir & 6 & Hypsodonthus pronaticornis & Saraç, 2003 \\
\hline Çandır & Ankara & 6 & $\begin{array}{l}\text { Hypsodontus pronaticornis } \\
\text { Tethytragus koehlerae } \\
\text { Turcocerus gracilus }\end{array}$ & Geraads, 2003 \\
\hline Ören-Kultak & Muğla & $3-5$ & Caprotragoides portvaricus & Saraç, 2003 \\
\hline Sofça & Kütahya & $7-8$ & Protoryx cf. carolinae & Sickenberg, 1975 \\
\hline Sinap & Ankara & $9-10$ & $\begin{array}{l}\text { Tethytragus koehlerae } \\
\text { Prostrepsiceros aff. vallesiensis } \\
\text { Prostrepsiceros elegans } \\
\text { Prostrepsiceros rotundicornis } \\
\text { Nisidorcas planicornis } \\
\text { Sinapodorcas incarinatus } \\
\text { Palaeoreas asiaticus } \\
\text { Protoryx solignaci } \\
\text { Pachytragus laticeps } \\
\text { Pseudotragus aff. capricornis }\end{array}$ & Gentry, 2004 \\
\hline Alçıtepe- İncirlikdere & Çanakkale & $9-12$ & Gazella sp. & Saraç, 2003 \\
\hline Çorakyerler & Çankırı & $10-12$ & $\begin{array}{l}\text { Oioceros rothi } \\
\text { Miotragocerus (Pikermicerus) sp. } \\
\text { Miotragocerus sp. } \\
\text { Tragoportax gaudryi } \\
\text { Tragoportax sp. } \\
\text { cf. Prostrepsiceros sp } \\
\text { Criotherium sp. } \\
\text { Majoreas cf. woodwardi } \\
\text { Gazella sp. } \\
\text { Plesiaddax inundatus } \\
\text { Protoryx sp }\end{array}$ & $\begin{array}{l}\text { Geraads,2013;Sevi } \\
\text { m } \\
\text { Erol,Tarhan,Mayda, } \\
\text { Kaya,Sönmez,Mutlu } \\
\text {, } 2016\end{array}$ \\
\hline Yukarı-Sazak & Denizli & $11-12$ & $\begin{array}{l}\text { Tragoportax amalthea } \\
\text { Protoryx carolinae }\end{array}$ & Oruç, 2009 \\
\hline Mahmutgazi & \multirow[t]{2}{*}{ Denizli } & 11 & $\begin{array}{l}\text { Tragoportax gaudry } \\
\text { Tragoportax amalthea } \\
\text { Protoryx sp. } \\
\text { Pseudotragus parvidens } \\
\text { Plesiaddax cf. inundatus } \\
\text { Gazella sp. } \\
\text { Palaeoreas lindermayeri } \\
\text { Oioceros wegneri } \\
\text { Palaeoryx pallasi }\end{array}$ & Geraads, 2017 \\
\hline Çal & & $11-12$ & $\begin{array}{l}\text { Gazella sp. } \\
\text { Palaeoryx pallasi } \\
\text { Palaeoreas sp. }\end{array}$ & Kaya, 2009 \\
\hline Çöpköy & Edirne & $11-13$ & Paleoryx pallasi & Saraç, 2003 \\
\hline
\end{tabular}




\begin{tabular}{|c|c|c|c|c|}
\hline Küçükçekmece & İstanbul & $10-11$ & $\begin{array}{l}\text { Bohlinia cf. attica } \\
\text { Gazella cf. ancyrensis } \\
\text { Majoreas cf. elegans } \\
\text { Prostrepsiceros sp. } \\
\text { aff. Protoryx cf. enanus } \\
\text { cf. Miotragocerus sp. }\end{array}$ & $\begin{array}{l}\text { Kostopoulos ve Şen, } \\
2016\end{array}$ \\
\hline Bayat & Kütahya & $11-12$ & $\begin{array}{l}\text { Tragoportax amalthea } \\
\text { Protoryx sp. } \\
\text { Gazella sp. } \\
\text { Prostrepsiceros elegans }\end{array}$ & Kaya, 2009 \\
\hline Hatunsaray-Kayadibi 1-3 & Konya & 11 & $\begin{array}{l}\text { Capra bihlini } \\
\text { Nisidorcas planicornis }\end{array}$ & $\begin{array}{l}\text { Sickenberg, } 1975 \\
\text { Saraç, } 2003\end{array}$ \\
\hline Şerefköy-2 & Muğla & 12 & $\begin{array}{l}\text { Gazella cf. capricornis } \\
\text { Palaeoryx pallasi } \\
\text { Sporadotragus parvidens } \\
\text { Skoufotragus cf. sp. schlosseri } \\
\text { Urmiatherium rugosifrons } \\
\text { Sinotragus sp. }\end{array}$ & $\begin{array}{l}\text { Karakütük ve } \\
\text { Kostopoulos, } 2015\end{array}$ \\
\hline $\begin{array}{l}\text { Yatağan-Salihpaşalar } 1 \\
\text { Karaağaç mevkii) }\end{array}$ & Muğla & 12 & $\begin{array}{l}\text { Gazella deperdita } \\
\text { Protoryx carolinae }\end{array}$ & Atalay, 1980 \\
\hline Yatağan-Elekçi & Muğla & 12 & $\begin{array}{l}\text { Gazella goudryi } \\
\text { Gazella deperdita } \\
\text { Palaeoryx pallasi }\end{array}$ & Atalay, 1980 \\
\hline Milas-Ulaş & Muğla & 12 & Tethytragus koehlerae & Saraç, 2003 \\
\hline Haliminhanı-Hayranlı & Sivas & $11-12$ & $\begin{array}{l}\text { Gazella capricornis } \\
\text { Tragoportax cf. amalthea } \\
\text { Protoryx sp. } \\
\text { Prostrepsiceros houtumschindleri }\end{array}$ & Bibi ve Güleç, 2008 \\
\hline Kemiklitepe & Uşak & $11-12$ & $\begin{array}{l}\text { Palaeoreas cf. elegans } \\
\text { Gazella sp. } \\
\text { Protoryx laticeps } \\
\text { Palaeoryx sp. } \\
\text { Oioceros wegneri } \\
\end{array}$ & Şen, 1994 \\
\hline Akkaşdağ1 & Kırıkkale & 12 & $\begin{array}{l}\text { Gazella aff. pilgrimi } \\
\text { Prostrepsiceros rotundicornis } \\
\text { Miotragoceros valenciennesi } \\
\text { Pachytragus crassicornis } \\
\text { Tragoportax aff. amalthea } \\
\text { Palaeoryx majori }\end{array}$ & Kostopoulos, 2005 \\
\hline
\end{tabular}

Anadolu' daki lokaliteler ve buradan çıkan bovid türleri.

Tablo 1'de Anadolu'da 16 ilde 22 lokalitede yapılan çalışmalardan elde edilen bovid türlerine ait toplanan veriler MN (Mammal Neogene) zonları dikkate alınarak kronolojik sıraya konulmuştur. $\mathrm{Bu}$ verilere göre Anadolu'da 53 farklı bovid türü belirlenmiştir.

Yunanistan'daki otuz farklı lokalitede tespit edilen bovid tüleri Tablo 2' de verilmiştir.

Tablo 2

Yunanistanda Bulunan Lokalitelere Ait Türler

\begin{tabular}{llll}
\hline Lokalite & $(\mathrm{MN})$ & Türler & Araştırmac1 \\
\hline & & $\begin{array}{l}\text { Dystychoceras macedoniensis } \\
\text { Tragoportax sp }\end{array}$ & \\
Dytiko & $\begin{array}{l}\text { Protragelaphus theodori } \\
\text { Palaeoreas lindermayeri } \\
\text { Gazella deperdita }\end{array}$ & Bouvrain, 2007 \\
& Palaeoryx sp. &
\end{tabular}




\begin{tabular}{|c|c|c|c|}
\hline Nikiti 1-2 & $8-9$ & $\begin{array}{l}\text { Tragoportax amalthea } \\
\text { Miotragocerus sp. } \\
\text { Gazella cf. capricornis } \\
\text { Gazella pilgrimi } \\
\text { Nisidorcas planicornis } \\
\text { Paleoreas lindermayeri } \\
\text { Palaeoryx cf. pallasi }\end{array}$ & $\begin{array}{l}\text { Kostopoulos, } \\
2016\end{array}$ \\
\hline Antonios & $4-5$ & Eotragus sp. & \multirow{12}{*}{ Koufos, 2006} \\
\hline Thymiana & 5 & $\begin{array}{l}\text { Tethytragus cf. kohlerae } \\
\text { Hypsodontus cf. gaopense }\end{array}$ & \\
\hline Pentalophos 1 & $9-10$ & $\begin{array}{l}\text { Ouzocerus pentalophosi } \\
\text { Helladorcas geraadsi } \\
\text { Gazella sp. } \\
\text { Protoryx sp. }\end{array}$ & \\
\hline Xirochori 1 & 10 & $\begin{array}{l}\text { Ouzocerus sp. } \\
\text { Samotragus praecursor } \\
\text { Palaeoryx sp. } \\
\end{array}$ & \\
\hline Ravin de la Pluie & 10 & $\begin{array}{l}\text { Mesembriacerus melentisi } \\
\text { Samotragus praecursor } \\
\text { Prostrepsiceros vallesiensis }\end{array}$ & \\
\hline Ravin des Zouaves 1 & 10 & $\begin{array}{l}\text { Mesembriacerus melentisi } \\
\text { Ouzocerus gracilis } \\
\text { Samotragus praecursor }\end{array}$ & \\
\hline Ravin des Zouaves 5 & 11 & $\begin{array}{l}\text { Tragoportax rugosifrons } \\
\text { Palaeoreas zouavei } \\
\text { Nisidorcas planicornis } \\
\text { Prostrepsiceros zitteli } \\
\text { Prostrepsiceros rotundicornis } \\
\text { Prostrepsiceros axiosi } \\
\text { Gazella pilgrimi } \\
\text { Gazella sp }\end{array}$ & \\
\hline Vathylakkos 1-2-3 & 12 & $\begin{array}{l}\text { Tragoportax rugosifrons } \\
\text { Gazella pilgrimi } \\
\text { Nisidorcas planicornis } \\
\text { Prostrepsiceros zitteli } \\
\text { Palaeoreas lindermayeri } \\
\text { Protoryx sp. }\end{array}$ & \\
\hline Prochoma & 12 & $\begin{array}{l}\text { Tragoportax rugosifrons } \\
\text { Nisidorcas planicornis } \\
\text { Prostrepsiceros zitteli } \\
\text { Gazella pilgrimi } \\
\text { Protoryx sp. } \\
\text { Palaeoreas sp. }\end{array}$ & \\
\hline \multirow[t]{2}{*}{ Ravin $\mathrm{X}$} & \multirow[t]{2}{*}{12} & $\begin{array}{l}\text { Gazella sp. } \\
\text { Palaeoryx pallasi } \\
\text { Tragoportax aff. amalthea } \\
\text { Oioceros rothi } \\
\text { Prostrepsiceros sp. } \\
\end{array}$ & \\
\hline & & Nisidorcas planicornis & \\
\hline Mytilinii & 12 & $\begin{array}{l}\text { Miotragocerus vallencienesi } \\
\text { Tragoportax sp. } \\
\text { Palaeoryx major } \\
\text { Pseudotragus parvidens } \\
\text { Gazella sp. } \\
\text { Protoryx sp. }\end{array}$ & \\
\hline
\end{tabular}




\begin{tabular}{|c|c|c|c|}
\hline Perivolaki & 12 & $\begin{array}{l}\text { Nisidorcas planicornis } \\
\text { Prostrepsiceros aff. rotundicornis } \\
\text { Tragoportax rugosifrons } \\
\text { Pheraios chryssomallos } \\
\text { Miotragocerus cf. vallencienesi } \\
\text { Gazella pilgrimi } \\
\text { Gazella sp. } \\
\text { Protoryx sp. } \\
\text { Palaeoryx sp. } \\
\text { Palaeoreas sp }\end{array}$ & \\
\hline Halmyropotamos & 12 & $\begin{array}{l}\text { Protragelaphus skouzesi } \\
\text { Prostrepsiceros rotundicornis } \\
\text { Prostrepsiceros sp. } \\
\text { Palaeoreas lindermayeri } \\
\text { Tragoportax amalthea } \\
\text { Palaeoryx pallasi } \\
\text { Gazella sp. }\end{array}$ & \\
\hline Chomateres & 12 & $\begin{array}{l}\text { Tragoportax gaudryi } \\
\text { Palaeoreas lindermayeri } \\
\text { Prostrepsiceros rotundicornis } \\
\text { Gazella sp. }\end{array}$ & \\
\hline Pikermi & 12 & $\begin{array}{l}\text { Prostrepsiceros rotundicornis } \\
\text { Oioceros rothi } \\
\text { Protoryx carolinae } \\
\text { Protragelaphus skouzesi } \\
\text { Palaeoryx pallasi } \\
\text { Palaeoreas lindermayeri } \\
\text { Tragoportax amalthea } \\
\text { Tragoportax gaudryi } \\
\text { Gazella capricornis } \\
\text { Sporadotragus parvidens }\end{array}$ & \\
\hline Kerassia & $11-13$ & $\begin{array}{l}\text { Tragoportax cf. amalthea } \\
\text { Gazella sp. }\end{array}$ & \\
\hline Ano Metochi & 13 & $\begin{array}{l}\text { Prostrepsiceros woodwardi } \\
\text { Gazella sp. }\end{array}$ & \\
\hline Achladion & $11-13$ & $\begin{array}{l}\text { Tragoportax amalthea } \\
\text { Palaeoryx cf. pallasi }\end{array}$ & \\
\hline Alifakas & $11-13$ & $\begin{array}{l}\text { Tragoportax amalthea } \\
\text { Palaeoryx sp. }\end{array}$ & \\
\hline Pyrgos Vassilissis & $9-13$ & $\begin{array}{l}\text { Gazella deperdita } \\
\text { Gazella cf. gaudryi }\end{array}$ & \\
\hline Ravin Ar. & $11-13$ & $\begin{array}{l}\text { Gazella sp. } \\
\text { Palaeoryx pallasi } \\
\text { Tragoportax aff. amalthea } \\
\text { Oioceros rothi } \\
\text { Prostrepsiceros sp. } \\
\text { Pachytragus sp. }\end{array}$ & \\
\hline Rhodes & $9-13$ & $\begin{array}{l}\text { Palaeoryx pallasi } \\
\text { Palaeoryx aff. stutzeli }\end{array}$ & \\
\hline Samos Adası & $11-12$ & $\begin{array}{l}\text { Miotragocerus valenciennesi } \\
\text { Miotragocerus sp. } \\
\text { Tragoportax rugosifrons } \\
\text { Tragoportax sp. } \\
\text { Gazella cf. capricornis } \\
\text { Gazella pilgrimi } \\
\text { Gazella mytilinii } \\
\text { Majoreas sp. }\end{array}$ & Kostopoulos,2009 \\
\hline
\end{tabular}




\begin{tabular}{|c|c|c|c|}
\hline & & $\begin{array}{l}\text { Sporadotragus parvidens } \\
\text { Palaeoryx majori } \\
\text { Palaeoryx pallasi } \\
\text { Palaeoryx sp. } \\
\text { Protoryx capricornis } \\
\text { Skoufotragus schlosseri } \\
\text { Skoufotragus laticeps } \\
\text { Urmiatherium rugosifrons } \\
\text { Samotragus crassicrornis }\end{array}$ & \\
\hline Tanagra & $9-13$ & $\begin{array}{l}\text { Gazella deperdita } \\
\text { Gazella sp. } \\
\text { Tragoreas oryxoides } \\
\text { Prostrepsiceros houtumschindleri }\end{array}$ & \multirow[t]{2}{*}{ Koufos, 2006} \\
\hline Triada & $9-13$ & $\begin{array}{l}\text { Tragoportax amalthea } \\
\text { Palaeoryx pallasi }\end{array}$ & \\
\hline
\end{tabular}

Yunanistan'daki lokaliteler ve buradan çıkan bovid türleri.

Tablo 2'de Yunannistan'da 30 lokalitede yapılan çalışmalardan elde edilen bovid türlerine ait toplanan veriler MN (Mammal Neogene) zonları dikkate alınarak kronolojik sıraya konulmuştur. $\mathrm{Bu}$ verilere göre Yunanistan'da 52 farklı bovid türü belirlenmiştir.

İran'da iki farklı lokalitede tespit edilen bovid tüleri Tablo 3' te verilmiştir.

Tablo 3

Iranda Bulunan Lokalitelere Ait Türler

\begin{tabular}{|c|c|c|c|}
\hline Lokalite & $(\mathrm{MN})$ & Türler & Araştırmacı \\
\hline Maragheh & $11-12$ & $\begin{array}{l}\text { Gazella capricornis } \\
\text { Gazella cf. ancyrensis } \\
\text { Demecquenemia rodleri } \\
\text { Oioceros atropatenes } \\
\text { Oioceros rothii } \\
\text { Nisidorcas sp. } \\
\text { Prostrepsiceros houtumschindleri } \\
\text { Prostrepsiceros cf. vinayaki } \\
\text { Prostrepsiceros fraasi } \\
\text { Prostrepsiceros cf. rotundicornis } \\
\text { cf. Palaeoreas sp. } \\
\text { Palaeoryx sp. } \\
\text { Urmiatherium polaki } \\
\text { Miotragocerus cf. maius } \\
\text { Miotragocerus sp. } \\
\text { Tragoportax cf. amalthea } \\
\text { Samokeros minotaurus } \\
\text { Skoufotragus laticeps }\end{array}$ & Kostopoulos ve Bernor, 2011 \\
\hline Ivand & 12 & $\begin{array}{l}\text { Oioceros atropatenes } \\
\text { Gazella sp. }\end{array}$ & $\begin{array}{l}\text { Ataabadi, Mohammadalizadeh, } \\
\text { Zhang, Watabe, Kaakinen, Fortelius, } \\
2011\end{array}$ \\
\hline
\end{tabular}

İran'daki lokaliteler ve buradan çıkan bovid türleri.

Tablo 3 'te İran'da 2 lokalitede yapılan çalışmalardan elde edilen bovid türlerine ait toplanan veriler MN (Mammal Neogene) zonları dikkate alınarak kronolojik sıraya konulmuştur. Bu verilere göre İran'da 19 farklı bovid türü belirlenmiştir. 
Tablo 4

$\ddot{U}$ Ç Farklı Ülkede Ortak Bulunan Tür Bilgileri

\begin{tabular}{|c|c|c|c|c|}
\hline Türler & $\begin{array}{c}\text { Türkiye } \\
\text { Yunanistan }\end{array}$ & $\begin{array}{l}\text { Türkiye } \\
\text { İran }\end{array}$ & $\begin{array}{c}\text { İran } \\
\text { Yunanistan }\end{array}$ & Ortak \\
\hline Gazella capricornis & + & + & + & + \\
\hline Gazella deperdita & + & - & - & - \\
\hline Gazella goudryi & + & - & - & - \\
\hline Gazella pilgrimi & + & - & - & - \\
\hline Gazella sp. & + & + & + & + \\
\hline Miotragoceros sp. & + & + & + & + \\
\hline Miotragoceros valenciennesi & + & - & - & - \\
\hline Nisidorcas planicornis & + & - & - & - \\
\hline Oioceros rothi & + & + & + & + \\
\hline Palaeoreas lindermayeri & + & - & - & - \\
\hline Palaeoreas sp. & + & + & + & + \\
\hline Palaeoryx majori & + & - & - & - \\
\hline Paleoryx pallasi & + & - & - & - \\
\hline Palaeoryx sp. & - & - & + & - \\
\hline Prosrepsiceros rotundicornis & + & + & + & + \\
\hline Prostrepsiceros houtumschindleri & + & + & + & + \\
\hline Prostrepsiceros $s p$ & + & - & - & - \\
\hline Prostrepsiceros vallesiensis & + & - & - & - \\
\hline Protoryx carolinae & + & - & - & - \\
\hline Protoryx sp & + & - & - & - \\
\hline Pseudotragus parvidens & + & - & - & - \\
\hline Samotragus praecursor & + & - & - & - \\
\hline Skoufotragus cf. sp. schlosseri & + & - & - & - \\
\hline Skoufotragus laticeps & - & - & + & - \\
\hline Sporadotragus parvidens & + & - & - & - \\
\hline Tethytragus koehlerae & + & - & - & - \\
\hline Tragoportax amalthea & + & + & + & + \\
\hline Tragoportax gaudryi & + & - & - & - \\
\hline Tragoreas oryxoides & + & - & - & - \\
\hline Urmiatherium rugosifrons & + & - & - & - \\
\hline
\end{tabular}

Anadolu, Yunanistan ve İran bölgelerinde bulunan ortak bovid türleri.

Tablo 4'te Türkiye- Yunanistan, Türkiye-İran, İran -Yunanistan ve bu biyocoğrafik bölgeler arasında ortak bovid türleri incelenmiştir. Türkiye- Yunanistan arasında 28; Türkiye-İran arasında 8; İran- Yunanistan arasında 10 ve her üç biyocoğrafik bölge arasında ise 8 bovid türünün ortak olduğu tespit edilmiştir.

\section{Sonuç, Tartışma ve Öneriler}

Bölgesel açıdan iklimin nem ve kuraklık derecesi hakkında bir tahmin oluşturmak için (Fortelius vd., 2002; Fortelius vd., 2014) geliştirmiş olduğu eko-metrik analiz oldukça kullanışlı bir yöntemdir. Paleo-çevresel koşulların tahmininde kullanılan bu yöntem temel olarak memeli türlerine ait hypsodonti diş kategorilerinin NOW veri tabanındaki analizine dayanır (Fortelius, 2008). Brachydont, mesodont ve hypsodont diş morfolojisine sahip bovid türlerin toplam diş tacı yüksekliğinin faunadaki toplam tür sayısına oranıyla test edilir. Testin sonuçları habitatların nemlilik ve kuraklık dereceleri hakkında fikir vermektedir. (Fortelius vd.,2002; Fortelius vd., 2014) yapmış olduğu nem haritasında Erken Miyosen dönemde hypsodonti ortalamaları nemli ve ormanlık çevre koşullarının homojen bir dağılımını vermiştir. Vallesian döneme kadar sınırlı sayıda bovidea ailesine ait türler mevcutken, Geç Vallesian ve Erken Turolian ile birlikte bir artış söz konusudur. $\mathrm{Bu}$ durumun yaklaşık Messinian dönemine kadar devam etmiştir. Anadolu Orta Miyosen dönem Paşalar (Bursa) ve Çandır (Ankara) faunalarının yanında Mordoğan (İzmir) faunası biyocoğrafik olarak benzer bir örüntüye sahiptirler. Mesodont ve hypsodont türleri Orta Miyosen sonlarına doğru artış göstermiş̧ir. Sofça faunasına olan benzerlik Paşalar ve Çandır lokalitesinden farklı olarak daha 
heterojenik bir örüntüye sahiptir (Kaya, 2017). Sinap Geç Miyosen Anadolu (Sub-Paratetis) faunas1 Yunanistan (Tablo 2) ve İran (Tablo 3) faunaları ile az çok benzerlik içindedir. Bir bütün içinde bakılırsa Anadolu Geç Miyosen faunalarının birbirine benzer bir eğilim içinde oldukları görülür.

Anadolu bovid faunası Senozoik dönem boyunca gerçekleşen paleobiyocoğrafik jeolojik ve ekolojik değişimlere bağlı olarak farklılıklar gösterir. Güneyde Hint Okyanusu'nun ve batıda Akdeniz'in oluşması ile Pasifik ve Atlantik Okyanusu'na bağlantılarının kurulması Tetis denizinin yavaş yavaş küçülmesine yol açmıştır. Eosen dönem boyunca Tetis ve Kutup Denizi Ural Dağları'nın bulunduğu bölge üzerinden Turgay Kanalı ile buluşmuştur (Rögl, 1999). Turgay Kanalı kapanması ile Asya ile Avrupa arasında karasal bağlantı meydana gelmiştir. Böylece Bering Boğazı üzerinden Kuzey Amerika kökenli memeli türleri Asya ve Avrupa'ya göç edebilmişlerdir. Hindistan kıtasının Asya'ya çarpması ile birlikte Himalayalar'ın ve Tibet Platosu'nun yükselmeye başlaması özellikle Batı Avrasya'da Tetis'in çekilmesini tetiklemiş ve Batı Asya ile Avrupa'da karasallaşmasının artmasına yol açmıştır. Senozoik boyunca yeryüzünde iklimi giderek kuraklaşıp soğumaya başlamış ve deniz seviyesi düşmüştür. İklimin kuraklaşmasına bağlı olarak ormanlık alanlar yerini kurak iklim koşullarına adapte olan geniş otlaklık alanlara ve savanlara bırakmıştır (Harvey Pough, Janis, Heiser, 2014; Rögl, 1999). Alpin sıradağların kuzeyinde bir iç deniz olan Paratetis oluşmuş; güneyinde ise Tetis-Akdeniz'e dönüşmüştür.

Anadolu'nun jeomorfolojik evrimi karasal oluşum ve gelişimi bu dönem boyunca ilerleme sağlamıştır. Bu dönem içinde Anadolu çarpma, aşınım, kırılma ile son halini almıştır (Demisoy, 2002; Şengör ve Y1lmaz, 1981). Tibet Platosu'nun yükselmeye devam etmesi özellikle atmosferdeki nem dolaşımını, dolayısı ile muson yağmurlarının ritmini değiştirerek Paratetis Denizi'nin çekilmesinde önemli rol oynadığı düşünülmektedir. Buna bağlı olarak Miyosen dönemin başlarında özellikle Anadolu'nun karasal olarak konumlandığı bölge olan Sub-Paratetis biyocoğrafik bölgesinde karasal alanlar artmıştır (Rögl, 1999). Deniz geçitleri Miyosen dönem boyunca denizlerin ekolojisi ve biyokimyasındaki değişimler için önemli faktörlerlerdir (Palcu, Golovina, Vernyhorova, Popov, Krijgsman, 2017). Orta Avrasya'yı kapsayan Paratetis Denizi konfigürasyonunda ki değişiklikler Orta Miyosen paleocoğrafik evriminde baskın bir rol oynamıştır. Senozoik dönemde dünya tropikal benzeri ormanlarla ve bu tür habitatlarda yaşayan hayvan türleri ile kaplıydı. Günümüzde ekvator bölgelerinde bu tür ormanlar hala mevcuttur. Ancak diğer enlemlerdeki farklı habitat tiplerinin (1lıman ormanlar ve otlak araziler) yanı sıra çöl ve tundra gibi birçok yeni habitatların eklenmesiyle birlikte bovid türlerinde de büyük bir çeşitlilik meydana gelmiştir. (Harvey Pough vd., 2014; Şen, 2013).

Gazella türleri Neojen / Kuaterner memeli bölgelerinde çok yaygındırlar. Geç Miyosen'de Gazella Doğu Akdeniz'e muhtemelen Asya'dan gelmiş ve daha sonra Avrupa ve Afrika'ya kadar yayılmışlardır. Gazella Turolian dönemde Doğu Akdeniz bölgelerinde genel olarak tüm memeli lokaliteleride gözlemlenmiştir (Agusti ve Wenderlin, 1995; Morales, Ginsburg, Soria, 1986). Doğu ve Batı Akdeniz provinsindeki lokaliteler karşılaştırıldığında Gazella'nın bu provinsler arasında 0,5 milyon yıllık kısa bir diyakronisi (zaman içindeki evrimleri) olduğu görülmüştür. Turolian döneminde çeşitlilik kazanmış olan bir başka grup ise Ovibovini'nin grubudur. Bugün misk öküzleri (Ovibos moschatus ve Budorcas taxicolor) tarafindan temsil edilen bu hayvanlar, boynuz çekirdeği ve bu bölgelerdeki belirgin kemik kalınlaşması içeren baziyoccipital/atlas artikülasyonlarının özelleşmeleriyle evrimsel tarihi boyunca karakterize edilmişlerdir. Geç-Orta Miyosen dönemde Avrasya'da ortaya çıkmış olan diğer Bovid cinsi Boselaphine'lerdir. Çin'den İspanya'ya ve oradan da Afrika'ya kadar yayılım göstermişlerdir (Gentry, 1992; Kostopoulos, 2005). Turolian döneminde, Yunan-İran arasındaki bölgede Ovibovinler, Urmiatherium, Parurmiatherium, Criotherium, Budorcas, Plesiaddax ve Mesembriacerus gibi birkaç cins mevcuttur. En ilgi çekici olanlardan birisi Alcelaphini benzeri uzun bacaklara ve 150 kg'l1k büyük bir gövdeye sahip olan Plesiaddax'tır. Dişleri hypsodont ve sementlidir, muhtemelen yapraklar, otlar ve otları içeren sert bitkilere dayanan bir diyete sahiptirler. Plesiaddax'ın boynuzları kısa ve kafatasının arkasından lateral olarak ayrılmıştır. Diğer Turolian Ovibovini grubundan Mesembriacerus uzun ve ince boynuzları güçlü bir şekilde geriye doğru olup, yaklaşık 25 kg'lık ağırlıkta ve orta büyüklükte bir boyuta sahiptir. Muhtemelen daha fazla açık ormanlık alanlara adapte olmuş olmalıdır. Dişlerden yapılan mikro analizler yumuşak bitkilere dayalı bir diyete sahip olduğunu göstermiştir (Gentry, 1992; Kostopoulos, 2005). Pleistosen dönem 
Praeovibos ve Ovibos için olası bir ataya uyduğu düşünülmektedir. Sonuncusu, belki de en önemlisi ilkel keçilerdir (Caprinae subfamilyası, Caprini tribusu veya Hippotragini tribusu). Turolian dönemde Palaeoryx, Protoryx ve Pachytragus faunası sırasında ortaya çıkmıştır. Muhtemelen Vallesian dönemlerinde Tethytragus benzeri bir formdan evrimleşmiş olmalıdır ancak Tethytragus, Protoryx için olası bir ataya çok daha fazla uyduğu düşünülmektedir (Agust1 ve Anton, 2002; Gentry, 1992; Kostopoulos, 2005). Büyük olasilıkla Vallesian krizinde ve Turolian dönemde ormana adaptif formların yok olmasından sonra Yunanistan-İran biyocoğrafyasında maksimum yayılım göstermişlerdir.

Avrupa'daki Turolian faunasının doruk noktasında bitki örtüsünü bileşimini etkileyen önemli çevresel değişiklikler söz konusudur. Dünya ölçeğinde yapılan bir dizi 8 ila 7 milyon yıl önce palefosoil ve dental karbonatların izotopik bileşiminde önemli bir değişiklik olduğunu göstermiştir. $\mathrm{Bu}$ kayma C3 içerenen bitkilerinin yerini $\mathrm{C} 4$ içeren bitkilere bırakması bu değişimin çayırların ve otlakların Avrasya ve Afrika'ya yayılmasının bir göstergesi olarak yorumlanmıştır (Agustı ve Anton, 2002). Otlaklar ve otlakların karakteristiği olan diğer bitkiler (C4) kurak ortamların daha zor fizyolojik koşullara daha iyi adapte olmuşlardır. Yunanistan-Anadolu-İran biyocoğrafyasında açık ormanlık faunasının batıya doğru uzaması, daha kuru koşullar ve otlakların egemen olduğu biyomlara olan çevresel değişime yorumlanmıştır. Ancak Erken Turolian dönemdeki hayvanlar bu duruma dâhil değildirler (Cerling, Harris, Macfadden, Leakey, Quade, Eisenmann, Ehleringer, 1997; Morgan Kingston, Marino, 1994; Quade, Cerling, Bowman, 1989). Özellikle Yunanistan-İran biyocoğrafyasında ki Boselaphine Miotragocerus ve Tragoportax arasında olağanüstü bir çeşitlilik görülür. Turolian döneminde Akdeniz-Peritethyan bölgesinde (Kuzey Afrika ve İspanya'dan Türkiye ve Ukrayna'ya kadar) Gazella ve Boselaphine yaygınlaştı. Boselaphine'ler ve Gazella'lar dışında, Prostrepsiceros, Ouzoceros, Nisidorcas, Samotragus, Oioceros ve Hispanodorcas gibi olağanüstü çeşitlilikte spiral boynuzlu antiloplar da görülmüştür. Boynuzun şekli ve pozisyonunu temel olarak farklılık göstermektedir. Prostrepsiceros oldukça uzun boynuzları ve nispeten daha uzun bacaklara sahiptir. Dişler sert yapraklara ve bitkilere dayanan diyeti gösteren özellikler sergilemektedir. Prostrepsiceros muhtemelen çalılıkla birlikte açık ormanlıkları içeren kuru bölgede yaşayan hızlı bir koşucu ve iyi bir sıçrayıcıydı. Ouzoceros'a benzemekle birlikte daha dik boynuzlu bir yapıya sahiptir. Nisidorcas küçük bir antilop olup kısa bacakları ve boynuzunda bulunan daha az spiral aralıklarıla tanımlanabilir. Ekolojik olarak çalılıkların bulunduğu açık bir provinste yaşayan ve çeşitli açılardan bugünün gazellalarına benzeyen morfolojik özellikler sahiptir. Samotragus ve Oioceros'un kafataslarının morfolojisi de ceylanlarınkine benzerdir, ancak bu cinsin dişleri daha özelleşmiştir. Tahmini olarak çimlik alanlara ve yapraklara çok daha fazla uyum sağlamış olduğu anlaşılmaktadır. Bunun yanısıra uzun bacakları açık savanalarda koşmaya oldukça el verişlidir (Morgan vd., 1994; Agust1 ve Anton, 2002).

Avrasya ve Afrika arasındaki Neojen / Kuvaterner memeli göç dalgaları ve önemli memeli fauna azalmaları iklimsel ve tektonik değişimlere bağlıdır. Bir hayvanın büyüklüğü ve göç mesafesi pozitif ilişki içindedir. Büyük boyutlu bir hayvan, yaşam süresi boyunca uzun bir göç mesafesini kat edebilirken bir mikromemeli (örneğin bir kemirgen), aynı mesafeyi kapatmak veya önemli bir topografik bariyeri geçmek için genellikle daha fazla jenerasyona ihtiyaç duyar. Bu durum dikkate alınırsa bovid evrimi ile göç faktörü eş zamanlı olmak zorundadır. Memeli fosillerinin taksonomik olarak belirlenmesi memeli göçü ve dağılımı için önemli bir faktördür. Biyolojik ve paleontolojik bir taksonun tanımındaki farklılıklar fosillerin sistematiğinin belirlenmesini daha hassas kılar (Koufos, 2003; Koufos, Kostopoulus, Vlachou, 2005; Kostopoulos ve Soubise, 2018).

Miyosen dönem boyunca Anadolu memeli paleobiyocoğrafyası temel olarak üç farklı aşamada ele alınabilir. Anadolu bovid faunasının Erken Miyosende genel olarak Eski Dünya faunal yapısına benzer olduğu düşünülmektedir. $\mathrm{Bu}$ dönemde nemli ve ormanlık koşulların hâkim olduğu paleobiyocoğfya öngörülmektedir. Orta Miyosen'de karasal alanların ve memeli türlerin çoğalması açısından düşünüldüğünde Anadolu'da Erken Miyosen döneme göre daha uygun bir ekosistemin varlığı söz konusudur. Doğuda Çin'den batıda Avrupa'ya kadar uzanan orta enlemlerde yarı-tropik ya da tropik bir iklim hâkimdi. Anadolu'da bu dönemde taksonomik kompozisyon olarak birbirlerine benzeyen Paşalar ve Çandır faunaları literatürde oldukça iyi bilinmektedir. Geç Miyosen olarak 
tarihlendirilen Anadolu seviyelerinden elde edilmiş polen fosilleri (Akgün, Akay, Erdoğan, 2007; Kayseri-Özer, Sözbilir, Akgün, 2014) faunanın daha fazla açık alanların artışını gösteren bitki örtüsündeki belirgin çoğalmayı destekler niteliktedir. Orta ve Batı Avrupa ormanlık alanlarında yaşayan büyük memeli türlerinin sayılarındaki dikkate değer bir azalmayla karakterize edilen ve litratürde Vallesiyen krizi olarak bilinen bu faunal olayının Anadolu memeli faunaları üzerindeki etkisi henüz çok iyi anlaşılmamıştır. Geç Miyosen dönem sonunda meydana gelen Mesiniyen Krizi Akdeniz'in neredeyse tamamen kuruması Avrupa ve Kuzey Afrika arasında memeli hareketliliğini etkilemeştir. Bu olayı takiben Afrika ve Avrasya arasındaki memeli paleobiyocoğrafyasını önemli ölçüde etkileyen Sahra Çölü’nün oluşması çetin çevresel koşulları meydana getirmiştir.

Geç Turolian'ın başlangıcını belirleyen önemli bir faunal değişimi söz konusudur ve bu dönemin karakteristik bir özelliğidir. Kesin jeolojik yaşlandırma veya paleomanyetik yaş hesaplamalarının eksik olması Orta-Geç Turolian dönem sınırlarının ayrımını zorlaştırmıştır. Birçok memeli taksonları bu dönemin sonunda diğer taksonlarla yer değiştirmiş̧ir (Koufos, 2003; SchmidtKittler, 1995). Mevcut veriler Geç Miyosen döneme ait bazı biyozonları ayırt etmeyi mümkün kı1sada, bu biyozonların sınırlarının ayırımı hala tam olarak net değildir (Koufos, 2003). İleride yapılacak çalışmalar Orta-Geç Turolian dönem sınırlarının daha net ayrımını mümkün kılabilecektir.

Geç Miyosen dönemde yaklaşık 10 Milyon yıl önce hızlı bir şekilde bovidler çeşitlenir ve bu durum 70 yeni cinsin oluşmasına yol açmıştır (Prothero ve Foss, 2007; Prothero ve Schoch, 2002). Geç Miyosen yayılımı kısmen birçok bovid türünün açık otlak habitatlara iyi adapte olmasından kaynaklanmış olmalıdır. Anadolu'da bovid türleri yer yer nemli ve yer yer kurak karakterdeki subtropik orman örtüsünün yanında yazın yeşil savanaların ve bazen de tamamen kuruyan step vejetasyonunun yaygın olduğunu göstermektedir. Miyosen bovid faunası, Anadolu'da oldukça zengin olarak görülmektedir. Bovidlerin geliştirdikleri adaptasyonlar, evrimsel değişimler, yayılım alanları, beslenme çeşitliliği ve özellikle de diğer türlerle olan bağlantıları Anadolu biyokronolojisi ve paleoekolojisinin anlaşılması açısından çok önemlidir. Anadolu-İran-Yunanistan biyocoğrafik bölgesindeki lokalitelerden elde edilen ortak 8 farklı türde bovid (Gazella cf. capricornis, Gazella sp., Miotragoceros sp.,Oioceros rothi, Palaeoreas sp. Prostrepsiceros houtumschindleri, Prostrepsiceros rotundicornis, Tragoportax amalthea) bu lokaliteler arasında büyük ölçüde paleoekolojik ve paleobiyocoğrafik benzerlik olduğunu ortaya koymaktadır. Aynı zamanda bu türlerin Anadolunun kıtalar arası geçişte bir köprü görevini gördügünü göstermiştir. Türlerin adaptasyonu dikkate alınırsa Geç Miyosen dönemde daha çok açık savan tipi daha kuru koşullar ve otlakların egemen olduğu biyomlara olan değişimler söz konusudur. Bu dönem boyunca İran-Anadolu-Yunanistan arasındaki biyocoğrafik bölgenin düşünülenin aksine daha kompleks bir yapıya sahip olduğu muhakkaktır.

Açıklamalar: Bu çalışma Kırşehir Ahi Evran Üniversitesi, Sosyal Bilimler Enstitüsü 569494 nolu “Anadolu Miyosen Dönem Bovidlerinin Paleobiyocoğrafyası ve Paleoekolojisi” konulu tezden üretilmiştir.

\section{Kaynaklar}

Agusti, J. and Anton, M. (2002). Mammoths, sabertooths, and hominids. NewYork: Columbia University Press.

Agustí, J., and Werdelin, L. (1995). Influence of climate on faunal evolution in the quaternary of Europe. Acta Zoologica Cracowiensa, 38, 1-2.

Akgün F., Akay E. and Erdogan B. (2002). Tertiary terrestrial to shallow marine deposition in central anatolia: a palynological approach. Turkish Journal of Earth Sciences, 11(2), 127-160.

Ataabadi, M. M., Mohammadalizadeh, J., Zhang, Z., Watabe, M., Kaakinen, A. and Fortelius, M. (2011). Late Miocene large mammals from Ivand, Iran. Geodiversitas, 33(4), 709-729. 
Atalay, Z. (1980). Muğla-Yatağan ve yakın dolayı karasal neojeninin stratigrafi araştırması. Türkiye Jeoloji Китити Bülteni, 23, 93-99.

Bibi, F. and Güleç, E.S. (2008). Bovidae (Mammalia: Artiodactyla) from the Late Miocene of Sivas, Turkey. Journal of Vertebrate Paleontology 28, 501-519.

Bouvrain, G. and Bonis, L. (2007). Ruminants (Mammalia, Artiodactyla: Tragulidae, Cervidae, Bovidae) des gisements du Miocène supérieur (Turolien) de Dytiko (Grèce). Annales de Paléontologie, 93, 121-147.

Cerling, T. E., J. R. Harris, B. J. Macfadden, M. G. Leakey, J. Quade, V. Eisenmann and J. R. Ehleringer. (1997). Global vegetation change through the miocene/pliocene boundary. Nature, $389,153-58$.

Colbert, E. H. and Moralles M. (1991). Evolution of the vertebrates. New York: Wiley-Liss.

Demirsoy, A. (2002). Genel Zoocoğrafya ve Türkiye Zoocoğrafyası. Ankara: Meteksan.

Fortelius, M. (2008). Neogene of the old world database of fossil mammals (NOW). University of Helsinki http://www.helsinki.fi/science/now.

Fortelius, M., Eronen, J., Jernvall, J., Liu, LP., Pushkina, D., Rinne, J., Tesakov, A., Vislobokova, I., Zhang, Z., and Zhou, L., (2002). Fossil mammals resolve regional patterns of Eurasian climate change over 20 million years. Evolutionary Ecology Research. 4, 1005-1006.

Fortelius, M., Eronen, T. J, Kaya, F., Tang, H., Raia, P., and Puolamaki, K. (2014). Evolution of Neogene Mammals in Eurasia: environmental forcing and biotic interactions. Annu Rev Earth Planet Science, 42, 579-604.

Gentry, A. W. (2004). Ruminantia (Artiodactyla) in Geology and of the Miocene Sinap formation Turkey. NewYork: Columbia University Press.

Gentry, A. W., Rössner, G.E. and Heizmann., E. P. J. (1992). The Miocene Land Mammals of Europe. Munich: Pfeil.

Gentry, A.W. (1990). Ruminant artiodactyls of Paşalar, Turkey. Journal of Human Evolution, 19, 529550 .

Geraads, D. (2003). Ruminants, other than Giraffidae from the Middle Miocene Hominoid locality of Çandır, Turkey. Cour. Forsch.-Inst. Senckerberg. Frankfurt: 24.

Geraads, D. (2013). Large Mammals from the Late Miocene of Çorakyerler, Çankırı, Turkey. Acta zoologica Bulgarica, 65(3), 381-390.

Geraads, D. (2017). Late Miocene large mammals from Mahmutgazi, Denizli province, Western Turkey. Neues Jahrbuch für Geologie und Palaontologie, 284(3), 241-257.

Harvey Pough, F., Janis C.M. and Heiser J.B. (2014). Omurgalı yaşamı (Vertebrata life), Çev. Ed. Mustafa Sözen. Ankara: Nobel Akademi Yayınc1l1k.

Karakütük, S. and Kostopoulos, D. S. (2015). Late miocene bovids from Şerefköy-2 SW Turkey and their position within the sub-Paratethyan. Acta Palaeontologica Polonica, 60(1), 49-66.

Kaya, F. (2017). Anadolu'nun neojen dönem memeli paleobiyocoğrafyası ve paleoekolojisi. Kebikec İnsan Bilimleri İçin Kaynak Arastirmali Dergisi, 43, 157-176. 
Kaya, T. (2009). Batı (Denizli, Muğla) ve İç Anadolu'da (Kütahya) yeni bulunan memeli fosil yatakları üzerinde ön çalışma. İzmir: Türkiye Bilimsel ve Teknolojik Araştırma Kurumu 32-41.

Kayseri-Özer M. S., Sözbilir H. and Akgün F. (2014). Miocene palynoflora of the Kocaçay and Cumaovası basins: a contribution to the synthesis of Miocene palynology, palaeoclimate, and palaeovegetation in western Turkey. Turkish Journal of Earth Sciences, 23, 233-259.

Kostopoulos D. S. (2016). Palaeontology of the Upper Miocene vertebrate localities of Nikiti (Chalkidiki Peninsula, Macedonia, Greece). Geobios 49(1-2), 119-134.

Kostopoulos, D. S. (2005). The Bovidae (Mammalia, Artiodactyla) from the late Miocene of Akkasdag1, Turkey. Geodiversitas, 27(4), 747-791.

Kostopoulos, D. S. and Bernor R. L. (2011). The Maragheh bovids (Mammalia, Artiodactyla) systematic revision and biostratigraphic-zoogeographic interpretation. Geodiversitas, 33(4), 649-708.

Kostopoulos, D. S. and Soubise, J. (2018). Palaeoreas, Majoreas, and Stryfnotherium gen. nov. (Mammalia: Artiodactyla: Bovidae) from the Late Miocene of Greece. In Annales de Paléontologie, 104(3), 231-247.

Kostopoulos, D. S. and Şen, Ş. (2016). Suidae, Tragulidae, Giraffidae, and Bovidae. Geodiversitas, 38(2), 273- 298.

Kostopoulos, D.S. (2009). The late miocene mammal faunas of the Mytilinii Basin, Samos Island, Greece. Beiträge zur Paläontologie Wien, 31, 13-26.

Koufos, G.D. (2003). Late Miocene mammal events and biostratigraphy in the Eastern Mediterrenean, distribution and migration of tertiary in Eurasia. Deinsea, 10, 343-371.

Koufos, G.D., (2006), The Neogene mammal localities of Greece: faunas, chronology and biostratigraphy. Hellenic Journal of Geosciences, 4l(1), 183-214.

Koufos, G.D., Kostopoulus, D.S. and Vlachou, T.D. (2005). Neogene/Quaternary mammalian migration in Eastern Mediterrenean. Belgian Journal of Zoology, 135(2), 181-190.

Morales, J., Ginsburg, L., and Soria, D. (1986). Los Bovoidea (Artiodactyla, Mammalia) del Mioceno inferior de España: filogenia biogeografía. Paleontologia i evolució, 20, 259-265.

Morgan, M. E., J. D. Kingston. and Marino, B. D. (1994). Carbon isotopic evidence for the emergence of C4 plants in the Neogene from Pakistan and Kenya. Nature, 367, 162-65.

Okay, A. I. (2008). Geology of Turkey: a synopsis. Anschnitt, 21, 19-42.

Oruç, S. (2009). Denizli (Yukarısazak) Neojen memeli faunasının Bovıdae (Artıodactyla) fosilleri ve Biyokronolojik, Paleobiyocoğrafik ve Paleoklimatolojik anlamlart. Yüksek Lisans Tezi, Ege Üniversitesi, İzmir.

Palcu, D.V., Golovina L.A., Vernyhorova Y.V., Popov S.V. and Krijgsman W. (2017). Middle Miocene paleoenvironmental crises in Central Eurasia caused by changes in marine gateway configuration. Global and Planetary Change, 158, 57-71.

Prothero, D. R. and Schoch, R. M. (2002). Horns, tusks, and flippers: The evolution of hoofed mammals. Baltimore: Johns Hopkins University Press. 
Prothero, R. D. and Foss E. S. (2007). Evolution of Artiodactyla. New York: The John Hopkins University Press.

Quade, J., T.E, Cerling and Bowman, J. R. (1989). Development of asian monsoon revealed by marked ecological shift during the Late Miocene in northern Pakistan. Nature, 342, 163-64.

Rögl F. (1999). Mediterranean and Paratethys. Facts and hypotheses of an Oligocene to Miocene paleogeography (short overwiev). Geologica Carpathica, 50(4), 339-349.

Saraç, G. (2003). Türkiye omurgall fosil yatakları. MTA Rapor no: 10609, Ankara.

Schmidt-Kittler, N. (1995). International symposium on Mammalian Biostratigraphy and Palaeoecology of the European Paleogene. Mu Chner Geowissencheften Abhandlungen, 10, 1 311.

Sevim Erol, A., Yavuz, A. Y., Tarhan, E., Mayda, S., Kaya, T. T., Sönmez Sözer, Ç., Mutlu, H. (2016). 2015 yılı Çorakyerler kazısı 38. Kazı Sonuçları Toplantısı, 1, 479-497.

Sickenberg, O. (1975). Die Gliederung des höheren jungtertiars und altquartars in der Türkei nach vertebraten und ihre bedeutung für die internationale Neogen-stratigraphie. Geologisches Jahrbuch, 15, 1-167, Hannover.

Şen, Ş. (1994). Les gisements de mammifères du Miocène supérieur de Kemiklitepe, Turquie. Bulletin du muséum national dihistoire natürelle, Paris.

Şen, Ş. (2013). Dispersal of African mammals in Eurasia during the Cenozoic: Ways and whys. Geobios, 46, 159-172.

Şengör AMC and Yilmaz Y. (1981). Tethyan evolution of Turkey: A plate tectonic approach. Tectonophysics, 75, 181-241.

Tekkaya, İ. (1974). Türkiye'de yeni bulunan omurgalı fosiller ve fosil yatakları, MTA Dergisi, 83, $109-112$.

Van Dam, J. A., Alcalá, L., Zarza, A. A., Calvo, J. P., Garcés, M., and Krijgsman, W. (2001). The upper Miocene mammal record from the Teruel-Alfambra region, Spain. Journal of Vertebrate Paleontology, 21(2), 367-385. 


\section{Extended Abstract}

\section{Introduction}

Anatolia, which is located between Asia, Africa and European continents since the Neogene period, maintains its signifigance as a peninsula where traces of migration are traced. Due to the migration of some vertebrate mammal fauna belonging to paleontological studies from Europe to the east, they were found at higher levels than they are represented in Europe in Anatolia. The classification of fossil artiodactyller is one of the most difficult among mammalian groups. Although the taxonomy of artiodactyls is quite easy in current materials, many difficulties are encountered when it comes to extinct artiodactyls. It is not an easy criterion for extinct artiodactyls to be subdivided into non-ruminant as well as ruminant chewing and non-chewing relaxation. When countless families of extinct artiodactyls are added to this list, it appears that a higher classification is necessary for the logical and appropriate grouping of many families. Bovids are the most diverse group of ruminants. They are located in South America, other continents outside Australia and naturally in different habitats. Bovids live in many habitats, from deep rain forests to woodlands, savannah, deserts, mountains and artificial environments. They are also available in various forms, such as, large and small. There are approximately 158 extinct generations and 47 generations living today. The most characteristic feature of bovid systematics is their horns and they are easily found in fossil studies. Horn morphology and horn core with different shapes are vital in the diagnosis of extinct bovid species. The second most important systematic determinant after the horns is the teeth. Teeth are the best protected fossil materials due to their hard structure. The height of Herbivorous mammal molar crow is one of the best known eco-metric indicators. Different diets and teeth generate different degrees of tooth wear between species. In this context, considering the hypsodontic average of different tooth structures between bovid species, it can be seen that there is a strong geographic correlation between hypsodonty rainfall. In addition, skull structure, premaxilla and skeletal structures are other differences in bovid species and they are quite distinctive features and one of the most important materials of paleontological studies.

\section{Method}

Biostratigraphic analysis of bovid species obtained from 22 formations in Anatolia, 30 formations in Greece and 2 formations in Iran, which are dated to Miocene period, were made with paleobiogeographic and paleoecological data in the literature. The collected data were put into chronological order considering MN (Mammal Neogene) zones (Dam, Alcalá, Alonso Zarza, Calvo, Garcés, Krijgsman, 2001). The eco-metric analysis method (Fortelius, Eronen, Jernvall, Pushkina, 2002; Fortelius et al., 2014), which is based on NOW database used to estimate paleo-environmental conditions, is correlated with paleoecological data on the basis of three regions considering the horn, cranial and dental characteristics of the species.

\section{Conclusion and Discussion}

Anatolian mammalian paleobiogeography can be studied in three different stages during the Miocene period. Anatolian bovid faunas are thought to be similar to the Old World fauna structure in early Miocene. Paleobiogeogia is foreseen with humid and forested conditions this period. Considering the proliferation of terrestrial areas and mammal species in the middle Miocene, there is a more favorable ecosystem in Anatolia than the early Miocene period.The Anatolian bovid fauna differs depending on the paleobiogeographic geological and ecological changes occurring during the Cenozoic period. In the late Miocene period, bovids varied rapidly about 10 million years ago, which led to the formation of 70 new genera. Late Miocene extension could have been partly due to the welladapted adaptation of many bovid species to open grassland habitats. In Anatolia, bovid species show that, subtropic forest cover which is humid and dry in places and green savannas in summer and sometimes completely dry steppe grasses are common. Miocene bovid fauna is seen as rich in Anatolia. The adaptations developed by bovids, evolutionary changes, propagation areas, nutritional diversity and especially their connections with other species are very important for the understanding 
of Anatolian biochronology and paleoecology. The eight common bovid species (Gazella cf. capricornis, Gazella sp., Miotragoceros sp., Oioceros rothi, Palaeoreas sp. Prostrepsiceros houtumschindler, Prostrepsiceros rotundicornis, Tragoportax amalthea) obtained from the localities in Anatolia-Iran-Greece provine, reveal that there is a great deal of paleoecological and paleobiogeographic similarities between these localities. At the same time, these species have shown that Anatolia acts as a bridge between the continents. If the adaptation of the species is taken into consideration, in the late Miocene period there are more open savanna type drier conditions and changes to the biomes dominated by pastures. During this period, the bioprovensy between Iran, Anatolia and Greece, contrary to the thought, has a more complex structure. 